\title{
Resümee und Ausblick
}

Das weltpolitische Handeln Deutschlands und der USA erwies sich im 20. Jahrhundert für den weiteren Werdegang des jeweils anderen von entscheidender Bedeutung. Es waren drei Auseinandersetzungen globalen Ausmaßes gewesen, die im Verlauf des 20. Jahrhunderts nicht nur die deutsch-amerikanischen Beziehungen bestimmen, sondern auch die internationalen Machtverhältnisse völlig neu definieren sollten. In der ersten Jahrhunderthälfte hatten zunächst die beiden 1914 und 1939 von Deutschland ausgegangenen Weltkriege die Vereinigten Staaten in eine zunächst ungewollte Führungsrolle hineingedrängt. Beide Male sollte der amerikanische Kriegseintritt zum Sieg der antideutschen Allianzen beitragen. Mit dem vollkommenen Zusammenbruch des Dritten Reichs im Jahre 1945 verlor Deutschland schließlich seine Rolle als eigenständiger internationaler Machtfaktor. Doch damit nicht genug: Der schon kurz nach dem Ende des Zweiten Weltkrieges heraufziehende Kalte Krieg führte 1949 sogar zur staatlichen Teilung Deutschlands. Fortan fungierten die Vereinigten Staaten als Schutz- und Führungsmacht der westlichen Welt gegen die kommunistische Herausforderung des von der Sowjetunion dominierten Ostblocks. Die Bundesrepublik Deutschland ebenso wie die DDR blieben aufgrund ihrer geopolitischen und ideologischen Frontstellung als unselbständige Objekte weiterhin im Zentrum des weltpolitischen Geschehens. Für beide Weltmächte besaß die feste Einbindung des jeweils kontrollierten deutschen Teilstaates in die eigene Einflußsphäre absolute, wenngleich nicht immer offen deklarierte Priorität. Aus bundesrepublikanischer Perspektive resultierte hieraus eine äußerst enge politische, militärische, wirtschaftliche und kulturelle Anbindung an die westliche Führungsmacht. „Die Leitkultur des Westens, die für die Bundesrepublik die Maßstäbe für den Neuanfang nach 1945 setzte, war Amerika“. ${ }^{1}$

Um das Jahr 1900 war dieser kometenhafte Aufstieg der USA zur Weltmacht noch nicht absehbar gewesen. Vielmehr schien es kurzzeitig so, als könne nun „Deutschlands Jahrhundert“ (Raymond Aron) anbrechen. ${ }^{2}$ Mit Blick auf die kulturelle und wissenschaftliche Bedeutung des 1871 gegründeten Kaiserreichs in den gut vier Dekaden vor dem Ausbruch des Ersten Weltkrieges hat auch der renommierte deutschstämmige US-Historiker Fritz Stern feststellt: „Das Vorkriegsdeutschland genoß weltweites Prestige und eine unübertroffene, friedliche Ausstrahlung. Deutsche Wissenschaft und deutsche Universitäten wurden als vorbildlich betrachtet, wie auch deutsche Technik und Leistungsfähigkeit. " 3 Tatsächlich läßt sich das soeben skizzierte, wechselvolle Verhältnis zwischen den USA und Deutschland insbesondere auf dem kulturellen Teilgebiet von Universität und

1 So Heinz Bude: Vorwort, in: ders./Greiner: Westbindungen, S. 8.

2 Hier zitiert nach Fritz Stern: Deutschland um 1900 - und eine zweite Chance, in: Hardtwig/Brandt: Deutschlands Weg in die Moderne, S. 32-44, hier S. 32 (Zitat).

3 Ebd. Vgl. in diesem Zusammenhang auch ders.: Verspielte Größe. Essays zur deutschen Geschichte, München 1996. 
Wissenschaft exemplarisch nachvollziehen. Galt Deutschland noch während des 19. und frühen 20. Jahrhunderts als der international führende Wissenschaftsstandort, so fiel dieser Platz spätestens mit der Zäsur der Jahre 1933-1945 und dem sich hieran unmittelbar anschließenden Beginn des Kalten Krieges den Vereinigten Staaten zu. Diese bemerkenswerte Entwicklung mit dem Hauptaugenmerk auf die besondere amerikanische Vorbildfunktion seit 1945 aus westdeutscher Perspektive nachzuzeichnen, war primäres Ziel der vorliegenden Studie.

Die Wechselwirkungen zwischen dem amerikanischen und deutschen Hochschulsystem sind Teil eines mehr als 200jährigen interkulturellen Transferprozesses, der sich freilich nicht allein auf den Zeitraum nach 1945 beschränkt. Um die westdeutsche Ausrichtung auf die USA seit Gründung der Bundesrepublik in einen größeren historischen Kontext einzubetten, erschien zunächst ein Blick auf die deutsch-amerikanischen Hochschul- und Wissenschaftsbeziehungen der Zeit vor 1945 notwendig. So konnte in Kapitel 2 dargelegt werden, daß die klassische deutsche Universitätsidee während des 19. und frühen 20. Jahrhunderts einen nicht unbedeutenden Einfluß auf die Entwicklung von Universität und Wissenschaft in den Vereinigten Staaten ausgeübt hatte. Als kulturelle Katalysatoren fungierten hierbei in erster Linie die rund 9000 bis 10000 Amerikaner, die zwischen ca. 1800 und 1920 in Deutschland studiert hatten und ihre dort gesammelten Erfahrungen anschließend in die USA importierten. Ein erheblicher Teil dieser Studenten besetzte nach der Rückkehr wichtige akademische bzw. administrative Schlüsselpositionen an amerikanischen Hochschulen. Konkret schlug sich die amerikanische Auseinandersetzung mit dem deutschen Universitäts- und Wissenschaftssystem u. a. in der Übernahme des Seminar-, Labor- und Vorlesungsbetriebs sowie deutscher Forschungsmethoden und wissenschaftlicher Fragestellungen nieder. Ferner übte auf amerikanische Gelehrte die Idee der akademischen Freiheit einen großen Reiz aus. Die Folge war ein sich gegen Ende des 19. Jahrhunderts etablierendes neues Rollen- und Selbstverständnis der amerikanischen Professorenschaft, das sich dezidiert am Vorbild des in seiner Forschung und Lehre vergleichsweise freien deutschen Ordinarius orientierte.

Trotz dieser bedeutenden Einflüsse war von amerikanischer Seite niemals eine vollständige Adaption des deutschen Universitätssystems angestrebt worden. Im Vordergrund stand vielmehr die behutsame Integration einzelner, als besonders nachahmenswert erachteter Komponenten des deutschen Hochschulwesens in die bereits bestehenden, d. h. vornehmlich englisch geprägten Bildungsstrukturen. „So ist schließlich [...] die idealtypische amerikanische research university", wie es der Erlanger Politologe Jürgen Gebhardt treffend formuliert hat, „eine nach dem Vorbild der deutschen Universität des 19. Jahrhunderts reformierte Institution und als solche ein deutsch-amerikanischer Hybrid.“4

In der Tat besteht kein Zweifel daran, daß die vor allem während der zweiten Hälfte des 19. Jahrhunderts vorgenommene Integration deutscher Hochschul- und Wissenschaftselemente maßgeblich zur Transformation des traditionellen und ursprünglich auf reine Lehr- und Erziehungsfunktionen beschränkten amerikani-

${ }^{4}$ Jürgen Gebhardt: Einleitung: Jenseits von Humboldt - Amerika?, in: Breinig/Gebhardt/ Ostendorf: Das deutsche und das amerikanische Hochschulsystem, S.1-23, hier S.6. 
schen College in eine moderne Forschungsuniversität mitteleuropäischen $\mathrm{Zu}$ schnitts beigetragen hat. In diesem Zusammenhang unterstreicht Michael Heyman, bis 1999 amtierender Generalsekretär der Washingtoner Smithsonian Institution und ehemaliger Kanzler der University of California in Berkeley:

„Blickt man aus amerikanischer Perspektive auf die Anfänge, so haben wir Deutschland und auch England viel zu verdanken. Aus England kam die Konzeption einer engen Beziehung zwischen Lehrkräften und Studenten hinsichtlich klar umrissener Lehrgegenstände und der Bemühung, einen Typ des kultivierten, gebildeten und würdigen Graduierten heranzubilden. Aus Deutschland kam eine andere Konzeption, die sich weniger auf die Wissensvermittlung vom Lehrenden auf den Studenten konzentrierte, sondern eher auf der Vorstellung einer funktionellen Einheit zwischen Forschung und Lehre basierte. “5

Der mit der Anlehnung an deutsche Standards verbundene Aufholprozeß hatte in den USA bereits um das Jahr 1900 dazu geführt, daß zahlreiche amerikanische Universitäten in der Qualität von Forschung und Lehre gegenüber ihren kontinentaleuropäischen Pendants kaum noch zurückstanden. Zwar genoß der deutsche Universitäts- und Wissenschaftsbetrieb in den Vereinigten Staaten auch weiterhin ein unvermindert hohes Ansehen, doch hatte nach der Jahrhundertwende ein Studium in Übersee aus amerikanischer Perspektive deutlich an Attraktivität verloren.

Auf deutscher Seite ließ dieser drohende Bedeutungsverlust die Idee zu einem deutsch-amerikanischen Professorenaustausch entstehen. Im Jahr 1905 kam es zum Abschluß eines ersten Austauschabkommens zwischen den Universitäten Berlin und Harvard, dem nur ein Jahr später ein weiteres Abkommen zwischen Berlin und der New Yorker Columbia University folgen sollte. Für die deutschen Initiatoren bestand das eigentliche Ziel des Professorenaustauschs darin, die schwindende Anziehungskraft der deutschen Universitäten auf amerikanische Studenten und Wissenschaftler zu stärken und damit die kulturelle und wissenschaftliche Strahlkraft Deutschlands in den USA weiter auszubauen. Mit anderen Worten: Der Professorenaustausch wurde in Berlin als ein Instrument auswärtiger Kulturpolitik betrachtet und nur im nachgeordneten Sinne als Mittel wissenschaftlicher Kontaktpflege. Die erhoffte Wirkung blieb jedoch aus. Weder konnten mit Hilfe des Professorenaustauschs vermehrt amerikanische Studenten für ein Studium in Deutschland gewonnen werden, noch gelang es den entsandten deutschen Professoren, den Beziehungen zu den Vereinigten Staaten eine nachhaltig positive Note zu verleihen.

Daß man im Kaiserreich die pro-deutsche Stimmung in den USA völlig falsch eingeschätzt hatte, sollte sich nach Ausbruch des Ersten Weltkrieges 1914 zeigen. Der knapp ein Jahrzehnt zuvor initiierte Professorenaustausch kam sofort zum Erliegen. Und auch die aggressiv-nationalistisch eingefärbten Verlautbarungen deutscher Gelehrter bei Kriegsausbruch trugen zusätzlich das ihre dazu bei, das Ansehen des Wissenschaftsstandorts Deutschland in Übersee zu diskreditieren. Nun zeigte sich, daß die in der Vorkriegszeit oftmals beschworene Internationalität der Wissenschaft gegen den kriegsbedingten Nationalismus nicht bestehen

5 Michael Heyman: Betrachtungen zum amerikanischen Hochschulwesen: Ist das amerikanische System für Deutschland relevant?, in: ebd., S. 23-32, hier S. 25. 
konnte. Dennoch darf der deutsch-amerikanische Professorenaustausch, und hierin liegt trotz aller Mißverständnisse und Widrigkeiten beiderseits des Atlantiks dessen übergeordnete Bedeutung, als die eigentliche „Geburtsstunde“ einer sich in der Folgezeit stetig weiterentwickelnden Intensivierung der Wissenschaftsbeziehungen beider Länder angesehen werden.

Nach einer kurzen Phase des Stillstands gelang es in der Zwischenkriegszeit, die akademischen Kontakte zwischen Deutschland und den USA wiederzubeleben. Verständlicherweise bestand gerade in Deutschland ein vitales Interesse daran, die kriegsbedingte internationale Isolation der deutschen Wissenschaft schnellstmöglich wieder aufzubrechen. Dabei erwies sich der Anfang der 1920er Jahre initiierte deutsch-amerikanische Studentenaustausch als ein erster wichtiger Schritt in Richtung dieses Ziels. Dieser Austausch war zudem maßgeblich für die Gründung des Akademischen Austauschdienstes e.V. (AAD) im Jahre 1925, also dem Vorläufer des 1931 ins Leben gerufenen Deutschen Akademischen Austauschdienstes (DAAD), verantwortlich. Welcher besondere Stellenwert dem Studentenaustausch mit den Vereinigten Staaten aus deutscher Sicht zufiel, belegt ferner die Tatsache, daß sich im Zeitraum zwischen 1924 und 1938 rund die Hälfte aller deutschen Austauschstudenten für ein Studium an einer amerikanischen Hochschule entschlossen hatte. Umgekehrt stammte ebenfalls beinahe die Hälfte der im gleichen Zeitraum in Deutschland studierenden Ausländer aus den Vereinigten Staaten. Schließlich konnte gegen Ende der 1920er Jahre auch der 1914/15 abgebrochene Professorenaustausch zwischen beiden Ländern kurzzeitig reaktiviert werden. Dieser änderte allerdings nichts an der überwiegend nationalistisch-konservativen Prägung der deutschen Professorenschaft, die sich bekanntlich vielfach zur dezidierten Republik- und Demokratiefeindschaft steigerte.

Allen Annäherungen entgegenlaufend, bereitete die nationalsozialistische Machtübernahme im Frühjahr 1933 den eben erst wieder aufblühenden akademischen Kontakten zwischen beiden Ländern binnen weniger Jahre erneut ein jähes Ende. Der durch das NS-Regime erzwungene Massenexodus deutscher Wissenschaftler sowie dessen Bemühen um eine ideologische Gleichschaltung des Hochschulwesens markieren den absoluten Tiefpunkt in der jüngeren deutschen Universitäts- und Wissenschaftsgeschichte. Hatten die deutschen Universitäten selbst noch in den Jahren der Weimarer Republik zu den führenden der Welt gezählt, beendete das nationalsozialistische Treiben endgültig diesen Status. Wenn auch von geregelten deutsch-amerikanischen Wissenschaftsbeziehungen zwischen 1933 und 1945 kaum gesprochen werden kann, blieb die Entwicklung von Universität und Wissenschaft in beiden Ländern doch während dieser zwölf Jahre in gewisser Weise schicksalhaft miteinander verbunden. Ein Großteil der aus Deutschland vertriebenen Wissenschaftler emigrierte in die USA, wo diese z.T. eine enorme Bereicherung für die dortige Hochschul- und Wissenschaftslandschaft bedeuteten. Gleichzeitig investierten die Vereinigten Staaten nach dem Kriegsausbruch 1939 und insbesondere nach dem eigenen Kriegseintritt 1941 in einem bis dato ungeahnten Ausmaß in Forschung und Wissenschaft. Denkt man sich die akademischen Beziehungen zwischen Deutschland und den Vereinigten Staaten in den vergangenen 200 Jahren rückblickend als eine Art Pendelbewegung, so beschreibt die Ära des Dritten Reichs eindeutig die Phase des Umschwungs zugunsten der USA. 
Der verlorene Krieg und die anschließende Besetzung Deutschlands durch alliierte Truppen verlieh den deutschen-amerikanischen Beziehungen nach 1945 eine völlig neue Qualität. Die zunächst in Kapitel 3 untersuchte Frage nach den Grundlagen, der Entwicklung und Zielsetzung der amerikanischen Hochschulpolitik während der ersten Phase der Besatzungszeit von 1945 bis 1949 ist insofern von besonderer Relevanz, als der amerikanischen Besatzungsmacht in diesem Zeitraum die Möglichkeit zufiel, der künftigen Entwicklung des (west-)deutschen Hochschulsystems eigene Impulse zu verleihen. Allerdings existierten speziell in Washington bis kurz vor Kriegsende keine konkreten Vorstellungen darüber, wie die künftige Hochschulpolitik innerhalb der eigenen Besatzungszone bzw. im besiegten Deutschland gestaltet werden sollte. Aufgrund praktischer Erwägungen standen zunächst militärische, politische und ökonomische Fragen im Zentrum der amerikanischen Nachkriegsplanungen. Hochschulbelange spielten demgegenüber in den ersten Wochen und Monaten nach der deutschen Kapitulation eine eher untergeordnete Rolle. Der gesamte Bildungssektor wurde lediglich als Bestandteil einer umfassenden Reeducation-Politik betrachtet. Folgerichtig wurden nach dem amerikanischen Einmarsch die sieben Universitäten und vier Technischen Hochschulen innerhalb der US-Zone auf zunächst unbestimmte Zeit geschlossen.

Trotz des Mangels an einem hochschulpolitischen Master Plan bestand innerhalb der OMGUS-Hochschulabteilung an der mittel- wie langfristigen Notwendigkeit einer grundlegenden Reform des höheren Bildungswesens in Deutschland kein Zweifel. Aus amerikanischer Perspektive hatte mit dem Untergang des Dritten Reichs auch auf universitärer Ebene eine fatale Entwicklung ihr Ende gefunden, welche die vormals international höchst angesehene deutsche Wissenschaft diskreditiert und in eine völlige Isolation geführt hatte. Deshalb maß die amerikanische Militärregierung vor allem der ideologischen „Reinigung“ der Universitäten, sprich der Entnazifizierung des Lehrkörpers, zunächst oberste Priorität bei. Die schrittweise Wiedereröffnung der Universitäten seit Herbst 1945 markiert sodann einen Kurswechsel in der amerikanischen Hochschulpolitik. Auf amerikanischer Seite war binnen weniger Monate die Erkenntnis herangereift, daß den deutschen Hochschulen für die Aus- und Heranbildung künftiger politischer, wirtschaftlicher, wissenschaftlicher und kultureller Eliten eine strategische Schlüsselfunktion zufiel. Diese veränderte Sichtweise auf die demokratiepolitische Funktion der Universitäten verband sich mit einem aktiveren hochschulpolitischen Engagement der amerikanischen Besatzungsmacht. Zwar blieb es bei der amerikanischen Grundhaltung, die auf eine Selbstreform der deutschen Universitäten setzte, gleichwohl gingen die zuständigen OMGUS-Stellen seit 1946 dazu über, konkrete Reformkonzepte zu erarbeiten. Zusätzlich verstärkt wurde das amerikanische Engagement durch die parallel verlaufenden Entwicklungen in den übrigen drei Besatzungszonen, wo Briten und vor allem Franzosen und Sowjets eine deutlich konsequentere Hochschulpolitik betrieben.

Die von der ERAB bzw. ECRD erarbeiteten Kriterien für eine moderne deutsche Nachkriegsuniversität wiesen in zentralen Punkten deutliche Anleihen beim amerikanischen Hochschulsystem auf, das aus Sicht der beteiligten US-Experten als Idealmodell eines in eine demokratische Gesellschaft eingebetteten Hochschulwesens galt. Im Zentrum des amerikanischen Reformansatzes stand der durchaus 
komplexe Bereich der künftigen Universitätsverfassung. Der traditionelle Verfassungsaufbau, so die damalige Meinung der amerikanischen Hochschuloffiziere, habe während der NS-Zeit zur ideologischen Gleichschaltung der Hochschulen maßgeblich beigetragen. An Hand zeitgenössischer Dokumente konnte diesbezüglich herausgearbeitet werden, daß die amerikanischen Reformvorschläge u.a. vorsahen, die künftige Universitätsverwaltung durch die Einrichtung von Hochschulräten bzw. Kuratorien auf eine gesellschaftlich kontrollierte Basis zu stellen, um das distanzierte Verhältnis zwischen Hochschulen und Öffentlichkeit zu verbessern. Nach dem Vorbild amerikanischer „Boards“ sollten die geplanten Hochschulräte aus allen gesellschaftlich relevanten Gruppen gebildet werden. Ferner wurde angedacht, auch das Rektorenamt nach dem Vorbild amerikanischer Universitätspräsidenten umzugestalten. Dabei sollte das bis dato in der Regel einjährige Rektorat durch eine Verlängerung der Amtszeit auf mindestens vier bis sechs Jahre sowie durch entsprechende Kompetenzerweiterungen modifiziert und gestärkt werden. Das Ziel der amerikanischen Reformvorschläge bestand also darin, die Administration der deutschen Universitäten auf mehrere Säulen zu verteilen, um somit ein - wie es in einer ERAB-Stellungnahme hieß - „system of checks and balances" herzustellen. ${ }^{6}$ Neben der Frage nach einer effizienten und sich gleichzeitig gegenseitig kontrollierenden Hochschulverwaltung widmeten sich die amerikanischen Reformvorschläge auch dem inneren Aufbau der deutschen Universität. In diesem Zusammenhang wurde angeregt, die als besonders schwerfällig erachteten Fakultäten traditionellen Zuschnitts aufzulösen und durch kleinere, an das amerikanische Departmentsystem angelehnte Verwaltungs- und Facheinheiten zu ersetzen.

Insgesamt betrachtet kann jedoch für die vierjährige OMGUS-Phase, also vom Zeitpunkt der deutschen Kapitulation am 8. Mai 1945 bis zur Gründung der Bundesrepublik und der damit einhergehenden Umwandlung der Militärregierung in eine zivile Hohe Kommission (HICOG) am 12. Mai 1949, von keinem wirklich nachhaltigen Erfolg der amerikanischen Hochschulpolitik gesprochen werden. Hinsichtlich der ursprünglichen Intention, die eine Demokratisierung des deutschen Universitätswesens mit Hilfe einer grundlegenden Verfassungs- und Verwaltungsreform herbeiführen wollte, ist zu konstatieren, daß es zu einer praktischen Umsetzung der von amerikanischen Bildungsexperten ausgearbeiteten Reformvorstellungen nicht kam. Wie dargelegt werden konnte, behielten innerhalb der Kultusministerien, der Rektorenkonferenz und der Universitäten restaurative Kräfte die Oberhand. Die von amerikanischer Seite angeregten Verfassungs-, Verwaltungs-, und Strukturreformen wurden in der Regel als mit den deutschen Gegebenheiten unvereinbar abgelehnt. Der universitäre Neuanfang sollte statt dessen durch ein bewußtes Anknüpfen an die „große“, zwischen 1933 bis 1945 lediglich unterbrochene, deutsche Universitätstradition vollzogen werden. Dieser Wille zur Rückbesinnung war kennzeichnend für die damalige Haltung und das Selbstverständnis eines Großteils der deutschen Professorenschaft, unabhängig von oder nur in gewisser Weise verbunden mit ihrer jeweiligen politisch-ideologischen Ein-

6 IfZ, OMGUS 5/299-3/29, Some Ideas Concerning the Reform of the Universities (11.9.1946), S. 1. 
stellung. Damit erwiesen sich die Hochschulen - im Unterschied zu anderen Bereichen des gesellschaftlichen, kulturellen und politischen Lebens im Nachkriegsdeutschland - gegenüber Einflüssen von „außen“ als vorerst äußerst resistent. ${ }^{7}$

Der während der Besatzungszeit von den Amerikanern hochgehaltene Gedanke einer Selbstreform der deutschen Universität nahm zwar Rücksicht auf die besondere historische Entwicklung des deutschen Universitätswesens und förderte zudem auf deutscher Seite auch das Vertrauen in demokratische Spielregeln. Gleichzeitig beraubte sich die US-Besatzungsmacht mit dieser Haltung jedoch der Möglichkeit, ihre eigentlich als notwendig erachteten Reformvorstellungen auch gegen deutsche Widerstände durchzusetzen. Hinzu trat noch ein weiterer, gleichfalls aus historischer Perspektive nicht zu unterschätzender Faktor: Die unter damaligen amerikanischen Bildungsexperten immer noch fest verankerte Hochachtung vor den Grundprinzipien der klassischen deutschen Universitätsidee, der - und dies war Persönlichkeiten wie Hartshorne, Karsen, Paty und Cottrell stets gegenwärtig - gerade die amerikanische Hochschulentwicklung viel zu verdanken hatte. Diese Gemengelage hemmte durchgreifende Reformen von außen wie von innen. Schließlich verschwand das Thema Verfassungsreform infolge des sich seit 1947/48 zuspitzenden Ost-West-Konflikts, der Gründung der Bundesrepublik und dem damit einhergehenden Wechsel von OMGUS zu HICOG im Mai 1949 von der amerikanischen Agenda. Mit dem Inkrafttreten des Besatzungsstatuts ging die Kontrolle des gesamten Bildungssektors an die zuständigen Stellen in der Bundesrepublik über. Damit waren die direkten amerikanischen Einflußmöglichkeiten auf die deutschen Hochschulverwaltungen und Universitäten fortan weitestgehend eingeschränkt.

Eine Gesamtbeurteilung der amerikanischen Hochschulpolitik während der OMGUS-Phase muß dennoch komplexer ausfallen. Trotz des aus hochschulpolitischer Perspektive primär restaurativen Charakters der Besatzungszeit - und hierin liegt deren eigentliche Bedeutung für die spätere Entwicklung von Universität und Wissenschaft in der Bundesrepublik - wurden in diesem Zeitraum durch die Vermittlung der amerikanischen Besatzungsmacht erstmalig Elemente des amerikanischen Universitäts- und Wissenschaftssystems, wie beispielsweise die Präsidialverfassung, das Departmentmodell oder der Hochschulratsgedanke, in den hochschulpolitischen Reformdiskurs eingeführt, die dann wenige Jahre später unter freilich andersartigen Umständen - den Reformprozeß der 1960er und 1970er Jahre abermals dominieren sollten.

Im Rahmen des sich anschließenden vierten Hauptkapitels wurde zunächst der Frage nachgegangen, inwieweit amerikanische Reformanregungen der Besatzungszeit in den westdeutschen Hochschulreformdiskurs der fünfziger Jahre hineingewirkt haben. Insbesondere die Dekade zwischen 1950 und 1960 veranschaulicht die ambivalente Grundhaltung, mit der man auf deutscher Seite diesem Thema begegnete: Einerseits wurde infolge der nach Kriegsende stetig steigenden Studentenzahlen und den damit einhergehenden neuen Anforderungen an die Universitäten die Notwendigkeit von Reformen immer deutlicher erkannt. Andererseits

7 Stucke: Mythos USA, S. 120. 
aber sollten mögliche Neuerungen nur äußerst behutsam, d.h. innerhalb der bestehenden Strukturen umgesetzt werden. Hinzu kam, daß der materielle Wiederaufbau des Hochschulwesens zu diesem Zeitpunkt immer noch absolute Priorität besaß. Forderungen nach weiterreichenden Veränderungen auf Verfassungs- bzw. Verwaltungsebene, so wie sie von amerikanischer Seite eingebracht worden waren, spielten demzufolge in den frühen fünfziger Jahren noch kaum eine nennenswerte Rolle.

Gleichwohl konnte am Beispiel maßgeblicher hochschulpolitischer Konferenzen und Stellungnahmen herausgearbeitet werden, daß entgegen dem vorherrschenden Rückbesinnungstrend der Reformdiskurs der fünfziger Jahre durchaus von Themen mitbestimmt wurde, die sich im Kern auf anglo-amerikanische Anregungen der Besatzungszeit zurückführen lassen. Im Vordergrund standen dabei vor allem folgende Aspekte: Die Einführung eines allgemeinbildenden Studium Generale, die Übernahme des angelsächsischen College-Systems, die Etablierung von Hochschulräten, der Auf- und Ausbau demokratiepolitisch relevanter Disziplinen wie der Sozial-, Politik- und Amerikawissenschaft und die Öffnung der Hochschulen für Studenten aus sozial schwächeren Bevölkerungsschichten. Daß der Reformprozeß in diesen Bereichen auch während der HICOG-Periode (1949-1955) von amerikanischer Seite aktiv vorangetrieben und unterstützt wurde, verdeutlicht nicht zuletzt der Umstand, daß wichtige Hochschulkonferenzen, wie beispielsweise die Weilburger Arbeitstagungen (1950) oder die Konferenz von Hinterzarten (1952), unter maßgeblicher organisatorischer, personeller und finanzieller Beteiligung der amerikanischen Hohen Kommission veranstaltet worden waren.

Welche bleibenden Spuren haben die OMGUS- und HICOG-Jahre innerhalb des westdeutschen Hochschul- und Wissenschaftssystems letztlich hinterlassen? Obgleich im Zusammenhang mit den ausgehenden vierziger und anschließenden fünfziger Jahren bislang zu Recht von einer Restaurations- und Wiederaufbauphase gesprochen werden kann, steht gleichzeitig außer Zweifel, daß der Wiederaufbau der westdeutschen Universitäts- und Wissenschaftslandschaft nach 1945 ohne die enormen finanziellen und materiellen Hilfsleistungen speziell aus den USA kaum denkbar gewesen wäre. Gefördert wurde neben dem Neubau von Mensen, Studentenwohnheimen und Universitätskliniken in beinahe allen westdeutschen Hochschulstädten auch der Aufbau demokratiepolitisch relevanter Forschungsund Universitätsinstitute. Hinzu traten immense Sachmittelleistungen für die Verpflegung von Studenten und Professoren, Möbel zur Ausstattung von Hörsälen und Seminarräumen, technische Apparaturen für Laboratorien und Institute sowie großzügige Bücherspenden. Im Gegensatz zu den amerikanischen Bemühungen um eine Reform der deutschen Hochschulverfassung kann somit das materielle und finanzielle Engagement der USA für den Wiederaufbau der deutschen Universitäten als äußerst erfolgreich betrachtet werden.

Dem amerikanischen Engagement im Hochschulbereich lagen selbstverständlich auch eindeutig politisch motivierte Zielsetzungen zugrunde. In diesem $\mathrm{Zu}$ sammenhang lassen sich vor allem zwei Stoßrichtungen festmachen: Einerseits sollte den Deutschen nach den Erfahrungen der nationalsozialistischen Herrschaft und des verlorenen Krieges auch auf wissenschaftlicher Ebene künftig nicht nur 
das Prinzip der Demokratie, sondern eben auch die amerikanische Kultur in ihrer gesamten Komplexität nähergebracht werden. Hierbei handelte es sich um ein Ansinnen, das freilich noch deutlich in der Tradition der Reeducation-Politik stand. Andererseits aber galt es, die junge westdeutsche Demokratie im Zuge des sich stetig verhärtenden Ost-West-Konflikts mit wissenschaftlicher Hilfe gegen die kommunistische Herausforderung zu stärken und somit das westlich-demokratische, von den USA dominierte Gegenmodell zu stabilisieren. „Der Kalte Krieg war“, wie Stöver es formuliert hat,

„eine politisch-ideologische, ökonomische, technologisch-wissenschaftliche und kulturellsoziale Auseinandersetzung, die ihre Auswirkungen bis in den Alltag zeigte. [...]. Die Nichtvereinbarkeit der beiden Lager führte zudem in den einzelnen Gesellschaften zu Polarisierung, Annäherungen an die jeweils andere Seite blieben [...] verdächtig. Gerade darin wird deutlich, daß der Kalte Krieg eigentlich nur Kombattanten kannte - Teilnehmer auf dieser oder jener Seite. “8

Aus amerikanischer Perspektive fiel in dieser politisch-ideologisch höchst aufgeladenen Atmosphäre speziell dem Auf- und Ausbau einer westdeutschen Politikund Amerikawissenschaft eine zentrale Bedeutung zu. Beide Disziplinen kann man zwar nicht als bloße „Wissenschaftsimporte“ aus den USA abstempeln, doch erfolgte die institutionelle, personelle und wissenschaftstheoretische Entwicklung der sich innerhalb des westdeutschen Universitätssystems nach 1945 neu etablierenden Fächer während des gesamten Untersuchungszeitraums hindurch unter kaum zu überschätzenden amerikanischen Einflüssen.

Ebenfalls ein unmittelbares Resultat des Kalten Krieges war die Gründung der Freien Universität Berlin im Herbst 1948. Obgleich die Initiative zur Etablierung einer neuen Hochschule im Westsektor Berlins als Gegeninstitution zur kommunistisch vereinnahmten „alten“ Friedrich-Wilhelms-Universität primär von deutscher Seite ausgegangen war, steht auch in diesem Fall außer Frage, daß die Neugründung ohne ein sich über Jahrzehnte hinweg erstreckendes amerikanisches Engagement keine vergleichbar erfolgreiche Entwicklung genommen hätte. Die Gründungsintention und ihre besondere geopolitische Lage ließen die FU binnen weniger Jahre zu einem Symbol wissenschaftlicher Freiheit innerhalb der westlichen Welt avancieren, dessen langfristige ideelle, materielle und finanzielle Unterstützung einen wichtigen Eckpfeiler der amerikanischen Außen- und Deutschlandpolitik während des Kalten Krieges bildete. Zudem war von amerikanischer Seite das dezidierte Ziel verfolgt worden, über den Aufbau der FU zu einer modernen Modellhochschule Einfluß auf die Entwicklung von Universität und Wissenschaft in der Bundesrepublik auszuüben. Etwas zugespitzt formuliert: Was während der Besatzungszeit gegen massive deutsche Widerstände nicht durchgesetzt wurde, sollte fortan mittels der Strahlkraft der FU erreicht werden.

Unter Berücksichtigung verschiedener Gesichtspunkte widmete sich das folgende sechste Hauptkapitel der Bedeutung des akademischen Austauschs mit den USA für die Entwicklung von Universität und Wissenschaft in der Bundesrepublik bis zur Verabschiedung des ersten HRG 1976. Tatsächlich kann die Wirkmächtigkeit des akademischen Austauschs in diesem Kontext nicht hoch genug einge-

8 Stöver: Der Kalte Krieg, S. 9. 
stuft werden. Die unmittelbare Begegnung mit der amerikanischen Demokratie, Kultur und Gesellschaft sowie der Kontakt mit dem amerikanischen Universitätsund Wissenschaftssystem hinterließ bei der überwiegenden Mehrheit der deutschen Austauschteilnehmer einen prägenden Eindruck. Gleichzeitig beschleunigte das Wiederaufleben des akademischen Austauschs mit den zur westlichen Führungsmacht aufgestiegenen USA auch die Reintegration der spätestens seit 1939 isolierten deutschen Wissenschaft in die internationale Scientific Community.

Aus amerikanischer Perspektive wurde die Wiederaufnahme der akademischen Austauschbeziehungen mit Deutschland zuallererst als Teil einer großangelegten, d.h. internationalen Kulturaußenpolitik betrachtet. Wie zu erwarten stieß das amerikanische Austauschprogramm hierzulande auf reges Interesse. Gemäß den amerikanischen Intentionen eröffnete dieses nach den Schrecken des Krieges Hunderten westdeutschen Studenten und Wissenschaftlern völlig neue Perspektiven. Allein die Vorstellung, den eigenen begrenzten und lange Zeit durch die nationalsozialistische Propaganda ideologisierten Horizont im Rahmen eines mehrmonatigen Aufenthalts in Übersee zu erweitern, erschien für viele faszinierend. Zudem erhoffte man sich von einem Studium in den USA Vorteile für die eigene Ausbildung bzw. für den späteren akademischen oder beruflichen Werdegang.

Wichtige Meilensteine im Hinblick auf den weiteren Ausbau des akademischen Austauschgedankens waren neben dem Fulbright-Abkommen von 1952 das nur ein Jahr später abgeschlossene Deutsch-Amerikanische Kulturabkommen. Obgleich beide Vertragswerke primär dem ursprünglichen Gedanken der Völkerverständigung verpflichtet blieben, bot die nun festgeschriebene akademische Partnerschaft mit den Vereinigten Staaten der jungen Bundesrepublik die Möglichkeit, den Anschluß an den internationalen Wissenschaftsbetrieb zu finden bzw. sich auch mit den aktuellen wissenschaftlichen Entwicklungen in den USA vertraut zu machen. Wie herausgearbeitet werden konnte, zeigten die an amerikanischen Universitäten und Forschungseinrichtungen gesammelten Erfahrungen deutscher Studenten und Wissenschaftler unmittelbare Rückwirkungen auf die Entwicklung von Universität und Wissenschaft in der Bundesrepublik. So darf die schon in den frühen fünfziger Jahren einsetzende Abwanderung deutscher Wissenschaftler in die USA - der „Brain Drain“ - als ein unmittelbares Resultat des akademischen Austauschs angesehen werden. Gemeinsam mit den überwiegend positiven Berichten zurückkehrender Austauschstudenten und -wissenschaftler über die zumeist ausgezeichneten Arbeitsbedingungen und Berufsperspektiven in den USA trug das Phänomen des „Brain Drain“ seit Anfang der 1960er Jahre bei den in der Bundesrepublik für Hochschulbelange zuständigen Stellen zu einer deutlichen Sensibilisierung hinsichtlich der Defizite des eigenen Universitäts- und Wissenschaftssystems bei, was im Rahmen des westdeutschen Reformdiskurses wiederum eine wachsende Orientierung an den Verhältnissen in den USA mit sich brachte. Der akademische Austausch mit den Vereinigten Staaten hatte somit einen entscheidenden Anteil daran, daß im Anschluß an die Restaurations- und Wiederaufbauphase der 1950er Jahre fortan zentrale Elemente des amerikanischen Hochschulsystems, die bereits während der Besatzungszeit von amerikanischer Seite zur Diskussion gestellt worden waren, die westdeutsche Reformdebatte dominierten. 
Anhand einschlägiger zeitgenössischer Beiträge zum Thema Hochschulreform konnte im Rahmen des siebten Hauptkapitels dargelegt werden, in welch beachtlichem Umfang das amerikanische Hochschulsystem schließlich nach 1960, zwar nicht als alleiniges Patentrezept, aber zweifelsohne als ein in zentralen Bereichen nachahmenswertes Modell herangezogen wurde. Wie die meisten damaligen Autoren in ihren Stellungnahmen immer wieder deutlich zu machen versuchten, ging es hierbei nicht um eine vorbildgetreue Übernahme des US-Systems im Sinne einer Blaupause, sondern um die weitaus subtilere Frage, welche konkreten Elemente des amerikanischen Systems gewinnbringend in den westdeutschen Kontext transferiert werden könnten. Dem lag die weitverbreitete Ansicht zugrunde, daß es den Vereinigten Staaten nach 1945 am besten gelungen sei, Universität und Wissenschaft auf die Herausforderungen der Zukunft einzustellen. Gleichzeitig bot der Verweis auf die USA die Möglichkeit, eine eigenständige ideelle Neukonzeption des deutschen Hochschulwesens zu umgehen und sich statt dessen auf vermeintlich schnell umsetzbare Verwaltungs- und Strukturreformen nach amerikanischem Muster zu konzentrieren.

Hinsichtlich der entscheidenden Frage, welche Aspekte des amerikanischen Hochschulsystems im Zuge des westdeutschen Reformprozesses der 1960er und 1970er Jahre am meisten Beachtung fanden, konnten in Kapitel 8 insgesamt sechs Schwerpunkte herausgearbeitet werden. So wurde die Diskussion über die Notwendigkeit einer universitären Verwaltungsreform maßgeblich durch den Verweis auf die Führungsstrukturen amerikanischer Universitäten bestimmt. Das dortige Präsidialsystem avancierte binnen weniger Jahre zum Synonym für Modernität und Effizienz. Wie in den USA üblich, sollten die zu Großbetrieben mutierenden deutschen Hochschulen künftig ebenfalls von einer managerähnlichen Persönlichkeit geleitet werden. Der jährlich wechselnde „Professoren-Rektor“ traditionellen Zuschnitts erschien - wie von amerikanischer Seite bereits während der Besatzungszeit vergeblich moniert worden war - nun auch unter deutschen Hochschulreformern als nicht mehr zeitgemäß. Allerdings entsprach der sich Ende der 1960er Jahre in den verschiedenen Landeshochschulgesetzen herauskristallisierende Universitätspräsident deutscher Ausprägung im Ergebnis weitaus mehr einem gestärkten Rektor herkömmlicher Art als einem „Manager-Präsidenten“ im amerikanischen Sinne. Verantwortlich hierfür war in erster Linie der Umstand, daß sich - trotz aller gegenteiligen Bekenntnisse - letztlich weder die Universitäten noch die zuständigen Kultusministerien dazu durchringen konnten, eigene Kompetenzen an das neugeschaffene Präsidentenamt abzugeben.

Neben der Hochschulspitze stand vor allem die Binnenstruktur der deutschen Universitäten im Zentrum damaliger Reformbestrebungen. Wie zeitgleich in der Präsidentenfrage wurde der Diskurs auch in diesem Punkt maßgeblich von einem amerikanischen Vorbild dominiert, nämlich dem amerikanischen DepartmentModell. Das mit der angestrebten Neuorganisation der universitären Binnenstruktur verbundene Ziel sollte sein, die wegen ihres gewachsenen Fächerspektrums unübersichtlich gewordenen Fakultäten in handlungsfähigere, d.h. fachbezogene Einheiten zu zergliedern und damit entsprechende Synergieeffekte freizusetzen. Zudem entsprach die Organisationsform des Departments, mit seiner besonderen Betonung des „Team Work“, einer seit den 1950er Jahren zu beobachtenden 
Orientierung an Führungs- und Arbeitsmodellen amerikanischer Provenienz. Demgegenüber schien die traditionelle Organisation von Forschung und Lehre im Rahmen der traditionellen Fakultäten und Institute nicht mehr zeitgemäß, ja sogar für den Rückstand der deutschen Wissenschaft gegenüber den USA mitverantwortlich zu sein. Nachhaltig verstärkt wurde diese Einschätzung wiederum durch die Erfahrungsberichte deutscher Nachwuchswissenschaftler, die in den Vereinigten Staaten die Arbeitsbedingungen innerhalb amerikanischer Departments kennen und schätzen gelernt hatten. Gegen Ende der 1960er bzw. Anfang der 1970er Jahre kam es in Anlehnung an das amerikanische Department-Modell zu einer fast flächendeckenden Einrichtung von Fachbereichen bzw. Abteilungen an den westdeutschen Universitäten. Doch vergleichbar der Umstrukturierung der Universitätsspitze handelte es sich hierbei nur um eine halbherzige Teiladaption. Zwar wurden wesentliche Organisationsmerkmale des amerikanischen Vorbilds übernommen, nicht aber dessen Personalstruktur. Im wesentlichen blieb das Lehrstuhlprinzip und damit die streng hierarchische Gliederung des wissenschaftlichen Personals an den Hochschulen weitestgehend unangetastet. Von einem gleichberechtigt-kollegialen Miteinander, das als typisches Merkmal amerikanischer Departments in den Berichten akademischer USA-Reisender immer wieder positiv hervorgehoben wurde, konnte demzufolge nicht gesprochen werden.

Rückblickend sollte sich dieses Defizit als ein Haupthemmnis für die erfolgreiche Adaption des Departmentgedankens erweisen, war es doch gerade die Kluft zwischen der vergleichsweise unsicheren Stellung des wissenschaftlichen Nachwuchses einerseits und der verbeamteten Professorenschaft andererseits gewesen, die hierzulande dem Abwanderungstrend nach den USA seit den 1950er Jahren immer wieder neue Nahrung gegeben hatte. Während in Übersee eine leistungsorientierte Hochschullaufbahn (Tenure track) über den Assistant-, Associate- hin zum Full-Professor als Regelfall möglich schien, blieb die Zukunft eines promovierten oder auch habilitierten Nachwuchswissenschaftlers innerhalb des traditionellen deutschen Systems bis zu dessen Berufung auf einen Lehrstuhl ungewiß. Deshalb geriet auch die Habilitation als Grundvoraussetzung für die Erlangung einer Professur zusehends ins Fadenkreuz der Kritik. Es kann kaum verwundern, daß sich der reformorientierte Blick bundesdeutscher Hochschulexperten auch in der Nachwuchsfrage zusehends auf die Verhältnisse an amerikanischen Universitäten richtete, wo die Stellung des Assistant-Professors einen weitaus sichereren Einstieg in eine akademische Karriere zu bieten schien. Seit Mitte der 1960er Jahre wurde daher die Forderung nach Abschaffung der Habilitation und der Einführung einer „Assistenz-Professur“ in Anlehnung an den amerikanischen Assistant-Professor immer vehementer vorgetragen. Wenn auch die Assistenz-Professur in einigen Bundesländern tatsächlich eingeführt wurde, so blieb doch die mit dieser Maßnahme erhoffte Wirkung letztlich aus. Dies lag in erster Linie am Festhalten an der Habilitation mit der Folge, daß diese für die Berufung auf einen Lehrstuhl in der Praxis auch weiterhin die eigentliche Voraussetzung bildete. Ähnlich wie bei der Umstrukturierung der Universitätsspitze und der Fakultäten handelte es sich auch bei der Einführung des Assistenz-Professors lediglich um eine Teiladaption, ohne dessen Stellung innerhalb des westdeutschen Universitätsgefüges bzw. den damit verbundenen Laufbahncharakter an das amerikanische Vorbild anzugleichen. 
Im Unterschied dazu erwies sich die in Anlehnung an das amerikanische Konzept des "Sabbatical Leave“ vorgenommene Einführung des „Forschungs(frei) semesters" als ein höchst positiver Impuls für die weitere Entwicklung der universitären Forschung in der Bundesrepublik. In den Vereinigten Staaten hatte sich bereits seit den 1880er Jahren an immer mehr Universitäten die Gepflogenheit durchgesetzt, den Professoren in der Regel alle sieben Jahre ein „Sabbatical Year" zu Forschungs- und Weiterbildungszwecken einzuräumen. In Deutschland existierte demgegenüber bis in die 1960er Jahre hinein keine eindeutige Regelung, die einem Hochschullehrer eine vergleichbare Freistellung für eigene Forschungsprojekte garantierte. Allerdings gewann das Thema seit den frühen 1950er Jahren mit dem Wiederaufleben der Wissenschaftsbeziehungen zu den USA an Bedeutung. Dafür lassen sich zwei konkrete Ursachen festmachen: Zum einen wurden im Zuge der wissenschaftlichen Dominanz der Vereinigten Staaten längere Forschungs- und Fortbildungsaufenthalte in Übersee, die von ministerieller Seite entsprechend zeitlich und finanziell abgesichert werden mußten, für deutsche Wissenschaftler immer notwendiger. Zum anderen lernten die deutschen Gäste während ihres Aufenthalts an amerikanischen Universitäten die dort gängige Praxis des „Sabbatical Leave“ kennen und schätzen. Eine unmittelbare Rückwirkung dieser in den USA gesammelten Erfahrungen war, daß sich deutsche Professoren im Rahmen von Berufungs- bzw. Rufablehnungsverhandlungen zunehmend unter Hinweis auf die amerikanischen Gepflogenheiten des „Sabbatical Leave“ ein regelmäßiges Freisemester durch die jeweiligen Hochschulen bzw. Kultusministerien zusichern ließen. Auf diese Weise konnte sich die Gewährung von Forschungssemestern bereits Ende der sechziger Jahre an den meisten westdeutschen Universitäten als gängige Praxis durchsetzen und sich zu einem festen Bestandteil des wissenschaftlichen Lebens in der Bundesrepublik entwickeln.

Ein weiteres Hauptaugenmerk damaliger Reformbestrebungen galt dem nicht immer unproblematischen Verhältnis von Hochschule und Öffentlichkeit. Bereits in der Besatzungszeit war sowohl von amerikanischer wie englischer Seite die Einführung von Hochschulräten bzw. Kuratorien angeregt worden, die als Verbindungsglied zwischen Universität und Gesellschaft fungieren sollten. Vor allem bei den Universitäten war dieser Vorschlag während der Restaurations- und Wiederaufbauphase des deutschen Hochschulwesens auf breite Ablehnung gestoßen. Nach den Erfahrungen des Dritten Reichs befürchtete man eine Einmischung außeruniversitärer Kräfte in zentrale Hochschulbelange. Erst mit Beginn der Reformperiode um 1960 gewann der Hochschulratsgedanke wieder an Aktualität. Die Notwendigkeit in höchstem Maße kostenintensiver Reformvorhaben ließ es nun angebracht erscheinen, den dafür aufkommenden Steuerzahler verstärkt in universitäre Entscheidungsprozesse einzubeziehen. Als Vorbild dienten insbesondere die „Boards“ amerikanischer Universitäten. Allerdings unterschieden sich die gegen Ende der 1960er eingeführten Hochschulräte und Kuratorien in einem ganz zentralen Punkt von ihrem amerikanischen Vorbild, denn sie besaßen lediglich beratende Funktion. Wie schon in der Präsidentenfrage waren letztlich weder die Universitäten noch die zuständigen Ministerien gewillt, an die neu eingerichteten Hochschulräte wirkliche Kompetenzen zu übertragen. 
Parallel zur Diskussion über die Einführung von Hochschulräten war verstärkt die Frage aufgekommen, ob seitens der Universitäten eine offensivere Informationspolitik gegenüber der Öffentlichkeit betrieben werden müsse. Abermals fiel der Blick dabei auf die USA. Dort hatten die renommierten Forschungsuniversitäten bereits lange vor dem Zweiten Weltkrieg damit begonnen, eigene Public-Relations-Abteilungen einzurichten. Bei einem z. T. privat finanzierten Universitätssystem wie dem amerikanischen war (und ist) es für die Hochschulen von fundamentaler Bedeutung, die eigene Außenwahrnehmung entsprechend attraktiv und informativ zu gestalten, um private Geldgeber als Unterstützer zu gewinnen. In der Bundesrepublik stand man im Zuge des seit 1960 diskutierten und wenig später massiv betriebenen Ausbaus des Hochschulwesens insofern vor einer vergleichbaren Situation, als auch hierzulande die steuerzahlende Bevölkerung von der Zukunftsträchtigkeit staatlicher Investitionen im Bildungssektor durch gezielte Informationen über die Tätigkeit und Bedeutung der Hochschulen überzeugt werden sollte. Tatsächlich wurde in deutschen Medien immer wieder der Umstand bemängelt, daß es für Journalisten weitaus einfacher sei, über Forschungsergebnisse amerikanischer Universitäten zu berichten als über den Wissenschafts- und Lehrbetrieb an deutschen Hochschulen. Um dieses Defizit zu beseitigen, kam es daher seit Ende der 1960er Jahre an den meisten westdeutschen Hochschulen zur Einrichtung eigener Pressestellen, die sich fortan um eine professionelle Außenwirkung und Informationspolitik bemühten.

Im Rahmen des neunten Hauptkapitels konnte anschließend gezeigt werden, daß auch das universitäre Bibliothekswesen von der seit 1945 einsetzenden USAOrientierung betroffen war. Schon lange vor dem Zweiten Weltkrieg galten die großen amerikanischen National- und Universitätsbibliotheken als die modernsten und größten der Welt. Diese international herausragende Stellung des amerikanischen Bibliothekswesens war das Ergebnis eines rasanten, im letzten Drittel des 19. Jahrhunderts einsetzenden Modernisierungs- und Sammlungsprozesses. Im Unterschied zu ihren kontinentaleuropäischen Pendants handelte es sich bei den amerikanischen Universitätsbibliotheken in der Regel um große Zentralbibliotheken, innerhalb derer die nach fachlichen Gesichtspunkten geordneten Bücherbestände in direkter räumlicher Nachbarschaft zueinander lagen und dadurch für den Benutzer bequem erreichbar waren. Hinzu kam, daß dem Leser ein Großteil der von einem Zentralkatalog erfaßten Bestände durch das Prinzip der Freihandaufstellung (open shelf) unmittelbar zugänglich gemacht wurde, ein Service, den gerade europäische Besucher als äußerst benutzerfreundlich erachteten.

Ein völlig anderes Bild bot demgegenüber der traditionelle Aufbau des universitären Bibliothekswesens in Deutschland. Während in Übersee seit dem ausgehenden 19. Jahrhundert die Tendenz zu einem einstufigen, d. h. zentralisierten Bibliotheksmodell beobachtet werden konnte, hatte die zunehmende wissenschaftliche Spezialisierung und fachliche Ausdifferenzierung hierzulande ein mehrstufiges Bibliothekssystem hervorgebracht. Neben der eigentlichen Universitätsbibliothek, die sich vorwiegend auf die Sammlung von Universalliteratur wie z. B. Enzyklopädien oder Lexika beschränkte, existierten eine Vielzahl voneinander autonom agierender Instituts-, Seminar- oder Lehrstuhlbibliotheken. Ein den Gesamtbestand aller universitären Teilbibliotheken erfassender Zentralkatalog - wie in den 
großen amerikanischen Bibliotheken üblich - existierte in der Regel nicht. Die strukturellen, aber auch ökonomischen Nachteile eines derart unkoordiniert arbeitenden Bibliothekssystems lagen auf der Hand: Unnötige Mehrfachanschaffungen und eine räumliche Zersplitterung der Bücherbestände, die ein immer wichtiger werdendes interdisziplinäres Arbeiten erschwerte. Um diese Rückständigkeit aufzuholen, intensivierte sich seit ca. 1950 die Beschäftigung westdeutscher (Universitäts-)Bibliothekare und Architekten speziell mit dem amerikanischen Bibliothekswesen. Am Beispiel der Universitätsbibliotheken in Stuttgart und Frankfurt am Main konnte nachgezeichnet werden, daß zentrale Elemente des strukturellen und organisatorischen Aufbaus amerikanischer Hochschulbibliotheken in der jungen Bundesrepublik bereits gegen Ende der fünfziger Jahre für universitäre Bibliotheksneubauten nutzbar gemacht wurden. Einen weiteren Modernisierungsschub erhielt das universitäre Bibliothekswesen dann seit Mitte der 1960er Jahre im Zuge der Gründung neuer Hochschulen. Hierbei eröffnete das gleichfalls maßgeblich aus den USA entlehnte Konzept der Campusuniversität erstmals die Möglichkeit, den Bau von Universitätsbibliotheken ohne Rücksichtnahme auf bestehende Strukturen neu zu konzipieren. Das Resultat war eine räumliche und organisatorische Zentralisierung des Bibliothekswesens nach amerikanischem Muster.

Tatsächlich boten die Hochschulneugründungen der 1960er und 1970er Jahre der Entwicklung von Universität und Wissenschaft in der Bundesrepublik vollkommen neue Perspektiven. Dies galt, abgesehen von der den Neugründungen zugedachten Entlastungsfunktion, insbesondere im Hinblick auf das seit 1945 mehr oder weniger intensiv diskutierte Thema einer nachhaltigen Hochschulreform. Mit Beginn der sechziger Jahre wurden die geplanten Neugründungen zunehmend als Chance begriffen, die seit langem als notwendig erachteten Reformen auf Verwaltungs-, Struktur- und Personalebene modellhaft umzusetzen. Ferner sollte unter dem Dach der neuen Hochschulen der Gedanke eines engeren Miteinanders von Lehrenden und Lernenden verwirklicht werden. Dabei handelte es sich um eine durchaus ambitionierte Zielsetzung, die an die architektonische Gesamtkonzeption der neuen Universitäten entsprechend neue Anforderungen stellte. Im Fokus stand die von Hochschulreformern und Architekten gleichsam $\mathrm{zu}$ beantwortende Frage, wie sich die vorgesehenen Reformen in einer adäquaten architektonischen Gesamtplanung widerspiegeln könnten. In diesem Kontext fiel der Campusuniversität speziell amerikanischer Ausprägung eine wichtige Vorbildfunktion zu. Ebenfalls beeinflußt durch die Erfahrungsberichte westdeutscher Studenten und Wissenschaftler galt diese aus verwaltungstechnischen, wissenschaftsorganisatorischen, aber auch sozialen Gesichtspunkten als die ideale Form einer Universität der Zukunft. Es war die von Hans Werner Rothe 1960/61 verfaßte Denkschrift für eine Universitätsneugründung in Bremen, die erstmalig das amerikanische Campuskonzept in den deutschen Kontext transferierte. Obgleich der von Rothe für die Hansestadt entworfene Idealcampus selbst niemals zur Ausführung kam, avancierte dessen Denkschrift zur planungstheoretischen Vorlage für beinahe alle späteren Gründungskonzepte. Am Beispiel der Universitäten Bochum, Regensburg und Konstanz konnte nachgewiesen werden, in welch beachtlichem Ausmaß der aus den USA entlehnte Campusgedanke auf die Planung und architektonische Umsetzung dieser frühen Neugründungen - trotz aller 
Unterschiede in der jeweiligen architektonischen und planerischen Ausformung eingewirkt hat. Somit stellen die westdeutschen Campusuniversitäten der 1960er und 1970er Jahre eindrucksvolle Manifestationen einer Reformpolitik dar, deren erklärte Zielsetzung es war, durch die Übernahme einzelner Elemente und Charakteristika des amerikanischen Hochschulsystems Universität und Wissenschaft in der Bundesrepublik zu modernisieren. ${ }^{9}$

Aus der Retrospektive erscheint allerdings fraglich, ob dieses ambitionierte Ziel wirklich erreicht wurde. In der Regel fehlt den deutschen Campusuniversitäten die besondere soziale und kulturelle Atmosphäre ihrer amerikanischen Vorbilder. Ein amerikanischen Universitäten vergleichbares Campus-Leben existiert hierzulande kaum, insbesondere weil auf den Bau von Studentenwohnheimen auf dem Universitätsareal verzichtet wurde. Auf die dennoch bis heute anhaltende symbolische Bedeutung des Campus-Motivs im Rahmen der deutschen Auseinandersetzung mit dem amerikanischen Hochschulsystem hat Mitchell G. Ash zu Recht kritisch hingewiesen. „Kaum ein Artikel zu diesem Themenkreis“, so der in Wien lehrende Historiker,

„scheint ohne ein Bild von einem parkähnlichen amerikanischen Campus auskommen zu können. Der häufige Gebrauch solcher Klischeebilder zeigt die zentrale Bedeutung, die den großen amerikanischen Eliteuniversitäten in dieser Diskussion beigemessen wird. Das ständige Schielen auf Harvard, Stanford oder ähnliche Universitäten spiegelt Denkfehler auf allen Seiten der deutschen Diskussion wieder. Darin kommt das Wunschdenken derjenigen zum Ausdruck, die durch einen Verweis auf die amerikanischen Eliteuniversitäten ,mehr Elite" auch in Deutschland erreichen wollen."10

Welche Gesamtbilanz läßt sich aufgrund der soeben skizzierten Untersuchungsergebnisse ziehen? Es ist festzuhalten, daß die Vereinigten Staaten seit den fünfziger Jahren des 20. Jahrhunderts den für die weitere Entwicklung von Universität und Wissenschaft in der Bundesrepublik dominierenden Referenzpunkt bildeten. Speziell mit Beginn der eigentlichen Reformphase um 1960 fiel der westdeutsche Blick in zentralen Fragen der Universitäts- und Wissenschaftsorganisation auf die USA. Diese Ausrichtung mündete schließlich in dem Versuch, einige wichtige Elemente des amerikanischen Hochschulsystems in den deutschen Kontext zu transferieren, um das hiesige Hochschulwesen nach amerikanischem Vorbild zu modernisieren. Gemäß der einleitend angeführten Definition Philipp Gasserts kann somit von einer partiellen „Amerikanisierung“ des westdeutschen Hochschulsystems insbesondere seit den 1960er Jahren gesprochen werden.

9 Vgl. hierzu auch die folgende Einschätzung von Jarausch: Das Humboldt-Syndrom, S.68f: „Obwohl als Mittel zum Abbau der Überfüllung konzipiert, versuchten neue Institutionen in Bochum, Bielefeld, Bremen oder Konstanz eine intellektuelle Gemeinschaft von Lehrenden und Lernenden durch die Akzentuierung der Forschung wiederherzustellen. Ihre Fachbereichsstruktur schuf kleinere kohärentere Einheiten; ihre liberalen Verfassungen gaben den Studenten eine Stimme in den Entscheidungsprozessen; und ihre thematische Konzentration und flexiblere Binnenstruktur [...] erleichterten die interdisziplinäre Zusammenarbeit. Ihre Campus-Anlagen konzentrierten Forschung und Lehre auf angenehme Weise an einem Ort und ihre strukturierten Curricula sowie die engere Betreuung durch das Lehrpersonal verbesserten die Studienqualität."

10 Mitchell G. Ash: Bedeutet ein Abschied vom Mythos Humboldt eine „Amerikanisierung“ der deutschen Universitäten?, in: ders.: Mythos Humboldt, S.253-256, hier S. 254 (Zitat). 
Gleichzeitig steht allerdings als ein weiteres Ergebnis der Studie fest, daß die in Anlehnung an amerikanische Vorbilder eingeleiteten Reformmaßnahmen mehrheitlich nicht die eigentlich intendierten Wirkungen erzielt haben. Weder konnte sich die Präsidialverfassung als alleinige Verwaltungsform durchsetzen, noch gelang es, flächendeckend und langfristig an den Hochschulen departmentähnliche Strukturen aufzubauen. Zahlreiche Universitäten kehrten seit Ende der 1970er wieder zur - freilich modifizierten - Rektoratsverfassung sowie zur traditionellen Fakultäts- und Institutsgliederung zurück. Auch gelang es dem neueingeführten Assistenz-Professor nicht, die Lage des wissenschaftlichen Nachwuchses nachhaltig zu verbessern, also in der Bundesrepublik eine mit den USA vergleichbare Hochschullaufbahn zu verankern. Bereits wenige Jahre nach seiner Einführung verschwand der Assistenz-Professor wieder von der universitären Bildfläche. Ein ähnliches Schicksal ereilte die in den 1960er und 1970er Jahren installierten Hochschulräte. Allein der Einrichtung des Forschungssemesters sowie dem gleichfalls aus den USA entlehnten Gedanken einer professionellen universitären Öffentlichkeitsarbeit ist es gelungen, sich innerhalb des deutschen Hochschulsystems fest zu etablieren und weiterzuentwickeln.

Wie aber läßt sich dieser ambivalente Gesamtbefund erklären? Offenkundig vermochte man nicht, die als vorbildlich identifizierten amerikanischen Hochschulelemente wirkungsvoll in das deutsche System zu integrieren. In der Regel blieb es bei Teiladaptionen, die in ihrer deutschen Ausformung nur noch in begrenztem Maße wirkliche Gemeinsamkeiten mit den amerikanischen Vorbildern aufwiesen. Beispielsweise verfügte weder ein deutscher Universitätspräsident noch ein deutscher Hochschulrat über annähernd vergleichbare Kompetenzen eines amerikanischen. War deren besondere Stellung und Funktion innerhalb des nach privatwirtschaftlichen Kriterien aufgebauten amerikanischen Hochschulwesens durchaus angemessen, erwies sich die Übertragung in das staatliche Universitätssystem der Bundesrepublik als schwierig. Trotz aller gegenteiligen Bekenntnisse zeigten sich weder die zuständigen Kultus- und Finanzministerien noch die von den Reformen unmittelbar betroffenen Universitäten gewillt, eigene Kompetenzen an die neugeschaffenen Ämter und Gremien abzugeben. In der universitären Praxis war es diesen demzufolge überhaupt nicht möglich gewesen, die mit ihrer Einführung erhofften Effekte zu entfalten.

Vergleichbare Rezeptionsprobleme zeigten sich auch in der Departmentfrage. Zwar war es speziell an den Neugründungen gelungen, Struktur- und Organisationsprinzipien nach amerikanischem Muster zu übernehmen und die dadurch erhofften finanziellen wie materiellen Synergieeffekte teilweise zu erreichen, aber die nicht minder bedeutsame soziale Komponente amerikanischer Departments, d.h. die relative Gleichstellung des Lehrpersonals, wurde nur partiell berücksichtigt. Das traditionelle Lehrstuhlprinzip sowie der damit einhergehende hierarchische Aufbau der deutschen Hochschullehrerschaft blieb weitestgehend unangetastet. Aus diesem Grunde mußte auch der Versuch beinahe zwangsläufig scheitern, dem Hochschullehrerberuf durch die Einführung der Assistenz-Professur einen eigenen Laufbahncharakter - vergleichbar dem amerikanischen ,Tenure Track' zu verleihen. Eine hierfür zwingend notwendige Veränderung der Rahmenbedingungen, insbesondere durch die Abschaffung der Habilitation, wurde nicht vorge- 
nommen. Diese blieb zumeist auch weiterhin Voraussetzung für die Berufung auf einen Lehrstuhl bzw. zur Erlangung einer Lebenszeitprofessur. „In der Konsolidierungsphase der 1980er Jahre schließlich“, wie Jarausch über das Ende der Expansions- und Reformphase der 1960er und 1970er Jahre zutreffend bemerkt hat, „diente ein Ausweichen auf traditionelle Formen neben notwendigen Kurskorrekturen als ein Tarnmantel für die Restauration professoraler Macht und Prärogative." 11

Einen evidenten Beleg für die Wirkungslosigkeit bzw. die geringe Nachhaltigkeit der damaligen Reformpolitik bietet ein Blick auf die aktuelle Hochschulreformdebatte in der wiedervereinigten Bundesrepublik. Hier zeigen sich auffallende Parallelen zum Diskurs der 1960er und 1970er Jahre. Mit einer fast identisch anmutenden Argumentation werden Reformen in teilweise exakt den Bereichen auf Verwaltungs-, Struktur- und Personalebene eingefordert, die schon vor knapp 50 Jahren im Zentrum der damaligen Reformmaßnahmen standen. Erstaunlicherweise scheint das Bewußtsein für diese historischen Voraussetzungen weitestgehend verlorengegangen zu sein.

Am Beispiel der Ende der 1990er Jahre von Seiten des Bundes eingeführten Juniorprofessur läßt sich diese Entwicklung veranschaulichen. Wir erinnern uns: Seit den 1950er Jahren wurde die Abwanderungsbewegung in die Vereinigten Staaten mit wachsender Sorge beobachtet. Als Hauptursache für diesen „Brain Drain“ galten die vermeintlich besseren Karrierechancen an amerikanischen Hochschulen, während hierzulande der Aufstieg in den Hochschullehrerberuf durch die Hürde der Habilitation und kaum vorhandener Personalstellen als extrem unattraktiv empfunden wurde. Die deshalb in Anlehnung an den amerikanischen AssistantProfessor eingeführte Assistenz-Professur sollte Abhilfe schaffen. Fast ein halbes Jahrhundert später begründet das Bundesministerium für Bildung und Forschung in einer Informationsbroschüre die Einführung der Juniorprofessur wie folgt:

„Eine der Ursachen des berüchtigten ,Brain Drain', der Abwanderung von Nachwuchskräften ins Ausland, war stets die mangelnde Karriereperspektive in Deutschland. Während die ehemaligen Kommilitonen gerade mit der Habilitation begannen, konnten deutsche Forscher z. B. in den USA bereits nach Abschluß der Promotion als Professor selbständig forschen und lehren. Die Juniorprofessur ist der schnelle, zeitgemäße Weg zur Universitätsprofessur. Eine deutsche Alternative im weltweiten Wettbewerb um die Spitzennachwuchswissenschaftlerinnen und -nachwuchswissenschaftler von morgen. "12

Erneut dient somit der amerikanische Assistant-Professor als unmittelbares Vorbild für den deutschen Juniorprofessor. Und wie der frühere Assistenz-Professor läuft nun auch die neugeschaffene Juniorprofessur Gefahr, an den unzureichenden Rahmenbedingungen zu scheitern. Als einer der wenigen historisch informierten Beobachter des heutigen Hochschulreformprozesses hat Manuel J. Hartung 2004 in „Der Zeit“ auf diese ernüchternde historische Parallele hingewiesen:

„Den Juniorprofessoren fehlt die Perspektive; ob sie nach den sechs Jahren Professor sind oder quasiarbeitsloser Privatdozent ist ungewiss. Der etwa in den USA übliche tenure track, eine Spur zur Dauerprofessur, auf die man bei guter Leistung kommt, fehlt in Deutschland.

11 Jarausch: Das Humboldt-Syndrom, S. 74.

12 Bundesministerium für Bildung und Forschung: An unseren Hochschulen bewegt sich etwas. Antworten und Fragen zur Juniorprofessur, o. O., o. J. (2001), S. 4f. 
Zudem kritisieren einige Juniorprofessoren, dass sie zu wenig Mitarbeiter und Sachmittel haben, aber eine zu hohe Lehrverpflichtung, um sich in der Forschung profilieren zu können. [...]. Viele Juniorprofessoren haben den Untergang der Assistenzprofessoren aus den siebziger Jahren als Warnung vor Augen - diese waren den heutigen Juniorprofessoren sehr ähnlich und wurden mit dem selben Argument eingeführt. Es sollte Schluß sein mit der „Helotentätigkeit des vom Ordinarius abhängigen Assistenten`, wie es damals hieß. Die Assistenzprofessoren scheiterten allerdings schon nach kurzer Zeit an Arbeitsüberlastung und am Beharrungsvermögen der Hochschule. “13

Das Beispiel der Assistenz- bzw. Juniorprofessur verweist auf die grundsätzliche Problematik, die mit dem Transfer eines fremden Hochschulelements in den heimischen Kontext verbunden ist. Einer medizinischen Organtransplantation gleich sollte im Rahmen eines derartigen Transferprozesses stets darauf geachtet werden, wie der ausgewählte Transfergegenstand am optimalsten in das eigene System integriert werden kann, um den innerhalb des Vorbildsystems identifizierten Positiveffekt annähernd zu erreichen. Es ist sicherzustellen, und dies zeigen im Positiven wie im Negativen gerade die Erfahrungen aus den Reformen der 1960er und 1970er Jahre, daß Übernahmen aus den Vereinigten Staaten allein unter konsequenter Berücksichtigung dortiger und hiesiger Rahmenbedingungen erfolgen. Der bloße Hinweis auf die tatsächlich oder vermeintlich besseren Verhältnisse an amerikanischen Hochschulen reicht als solcher nicht aus, um mehr oder weniger tiefgreifende Veränderungen innerhalb des deutschen Hochschulsystems zu rechtfertigen. Auf die Gefahren eines derartigen Automatismus hat im Jahr $2000 \mathrm{zu}$ Recht auch der Deutsche Anglistenverband mit Nachdruck hingewiesen:

„Es ist unerfreulich, daß sich die Auseinandersetzungen um den besten Weg für die Reform der deutschen Hochschulen zunehmend auf die Forderung verengen, an Deutschlands Universitäten möglichst schnell nordamerikanische Verhältnisse zu schaffen. Diese Zielvorgabe wird aber nicht von statistisch belegten Untersuchungen abgeleitet, die eine relative Unterlegenheit der deutschen Universität beweisen könnten, sondern beruht auf einem oft gedankenlosen Wiederholen der unbegründeten Behauptung, das nordamerikanische Universitätssystem sei dem deutschen überlegen. Die Amerikanisierung der deutschen Universität wird also unter Mißachtung der tatsächlichen Verhältnisse in Nordamerika propagiert. “14

Horst Mewes, Professor für Politische Theorie und Philosophie an der University of Colorado in Boulder, hat als Kenner beider Systeme sogar grundsätzlich in Frage gestellt, ob die Übertragung einzelner amerikanischer Teilelemente überhaupt möglich sei:

„Als unhaltbar beurteile ich allerdings die weit verbreitete Meinung, daß man wenigstens bestimmte Elemente des amerikanischen Hochschulsystems nach Deutschland transferieren könne, wenn schon eine komplette Übernahme nicht möglich ist. Die Teile [...] dürfen

13 Manuel J. Hartung: Immer Ärger mit dem Junior, in: Die Zeit vom 5. 8.2004.

14 Vorbild Nordamerika? Zum problematischen Vergleich Nordamerikanisches/Deutsches Hochschulsystem. Stellungnahme des deutschen Anglistenverbandes, erstellt von Stephan Kohl in Zusammenarbeit mit Monika Fludernik und Hubert Zapf, Würzburg 2000, S.1. Vgl. zudem den Abdruck Stephan Kohl u. a.: Vorbild Nordamerika? Zum problematischen Vergleich Nordamerikanisches/Deutsches Hochschulsystem, in: Mitteilungen des Deutschen Germanistenverbandes 2/3 (2000), S.302-320. Vgl. auch Hubert Zapf: Vorbild Amerika? Anmerkungen zum Vergleich des deutschen und des amerikanischen Hochschulsystems, Augsburg 2007. 
nämlich von ihrer Funktion und Bedeutung her nicht von dem System als Ganzem getrennt werden: Kann Letzteres nicht imitiert werden, so können es ebenso wenig seine signifikantesten Teile. “15

Tatsächlich läßt sich in der Reformdiskussion der vergangenen Jahre oftmals eine Art Reflex beobachten, der beinahe in jeder Frage von hochschul- und wissenschaftspolitischer Relevanz auf die Verhältnisse in den Vereinigten Staaten verweist. ${ }^{16}$ Dabei wird allerdings nicht selten außer acht gelassen, daß beide Hochschulsysteme in ihrer heutigen Gestalt auf völlig unterschiedlichen historischen Entwicklungen aufbauen. Allein die Art der Hochschulfinanzierung ist nicht vergleich- und somit auch nicht einfach übertragbar. ${ }^{17}$ Die dennoch häufig gestellte Forderung nach einer stärkeren Privatisierung des deutschen Hochschulwesens, ja nach der Gründung reiner Privatuniversitäten, berücksichtigt daher kaum die andersartigen steuer- und stiftungsrechtlichen Gegebenheiten bzw. Traditionen in beiden Ländern. Keine deutsche Universität verfügt auch nur annähernd über das Stiftungskapital einer der führenden amerikanischen Privathochschulen. ${ }^{18}$ Auch bewegt sich die Einwerbung sogenannter Drittmittel hierzulande auf einem völlig anderen Niveau als in den Vereinigten Staaten üblich. Selbst so prominente deutsche Privatgründungen wie Witten-Herdecke und die International University Bremen sind und bleiben wohl auch auf unabsehbare Zeit auf massive staatliche Unterstützungsleistungen angewiesen. „Daß die beiden neuen Privatuniversitäten in Deutschland [...]“, wie es der in St. Louis lehrende Paul Michael Lützeler 2002 etwas zugespitzt in der Zeitschrift „Merkur“ ausgedrückt hat,

„ihr Gründungskapital von der jeweiligen Landesregierung erhielten, ist bezeichnend. Eine private Universität aus Mitteln der öffentlichen Hand finanziert? Das klingt wie ein Widerspruch in sich selbst. In den USA ist es gerade umgekehrt: Dort fließen immer mehr Mittel aus privater Hand in die Budgets der Staatsuniversitäten."19

Zudem sollte nicht vergessen werden, daß - abgesehen von den immer wieder ins Feld geführten Ivy-League-Institutionen - die meisten amerikanischen Hochschulen nicht das durchschnittliche Niveau einer deutschen Universität errei-

15 Horst Mewes: Das amerikanische Hochschulsystem - ein Modell?, in: Breinig/Gebhardt/ Ostendorf: Das deutsche und das amerikanische Hochschulsystem, S. 195-207, hier S. 197 (Zitat).

16 Vgl. exemplarisch Lüst: Laßt uns von Amerika lernen, in: Die Zeit vom 3.5.1996.

17 Vgl. Alan Geiger: Kontrolle und Finanzierung der amerikanischen Hochschulen: Das Beispiel Ohio, in: Breinig/Gebhardt/Ostendorf: Das deutsche und das amerikanische Hochschulsystem, S.33-44, sowie Hans N. Weiler: Kontrollgewalt und Finanzstrukturen des deutschen Hochschulwesens im Wandel, in: ebd., S. 45-66.

18 Vgl. Paul Michael Lützeler: Wissenschaftlicher Fortschritt durch Internationalität. Die amerikanische Universität als Modell, in: Merkur 11 (2002), S. 1047-1051; Rainer Künzel: Politische Kontrolle und Finanzierung - Die Zukunft staatlicher Steuerung, in: Ash: Mythos Humboldt, S.181-194.

19 Lützeler: Wissenschaftlicher Fortschritt durch Internationalität, S.1048. Vgl. diesbezüglich auch die Einschätzung zur Gründung der International University Bremen bei Meinolf Dierkes/Hans Merkens: Zur Wettbewerbsfähigkeit des Hochschulsystems in Deutschland, Berlin 2002, S. 24: „Ob die finanzielle Unterstützung von Filialgründungen US-amerikanischer Universitäten durch den deutschen Steuerzahler - wie in Bremen im Fall der Rice University geschehen - eine sinnvolle Strategie zur Erhöhung der Wettbewerbsfähigkeit des Bildungsstandorts Deutschland ist, bleibt abzuwarten.“ 
chen. ${ }^{20}$ Dieser Umstand kann freilich nicht zufriedenstellen, er zeigt aber, daß das amerikanische Hochschulsystem eben nicht als solches vorbildlich sein kann, sondern lediglich einige wenige Spitzenuniversitäten. ${ }^{21}$ Und gerade weil dem so ist, stellt sich die entscheidende Grundsatzfrage, inwieweit „Äpfel mit Birnen“ überhaupt nutzbringend verglichen werden können. ${ }^{22}$

In den vergangenen Jahren läßt sich aufgrund der Verknappung staatlicher Mittel auch unter deutschen Universitäten ein immer intensiver werdendes Bemühen beobachten, die Finanzierung von Forschung und Lehre auf eine zweite Säule zu stellen. ${ }^{23}$ Naturgemäß fällt dabei der Wirtschaft eine zentrale Rolle zu. So warb beispielsweise der damalige Rektor der Universität Regensburg, Helmut Altner, im Rahmen einer am 29. Mai 2001 unter dem Titel Princeton an der Donau? vor der Industrie- und Handelskammer Oberpfalz/Ostbayern gehaltenen Rede für die Unterstützung der Regensburger Universitätsstiftung. Mit Blick auf die USA sagte Altner:

„Mehr Dynamik ist gefragt. Ein vertrauensvolles, der Staat wird's schon richten', reicht nicht aus. Wer Spitzenleistungen will, muß selbst Hand anlegen! [...] Um strukturelle Vorteile gegenüber konkurrierenden Universitäten zu erreichen, bedarf es auch eines massiven und gezielten Mäzenatentums. Dafür möchte ich Sie werben. Dabei plädiere ich für amerikanische Verhältnisse in der Oberpfalz. “24

Neben dem Versuch, das deutsche Hochschulwesen durch Privatgründungen oder die verstärkte Einwerbung von Drittmitteln an die Spitzen der amerikanischen Universitätsliga aufschließen zu lassen, hat unter derselben Zielsetzung im Spätsommer 2004 der Vorschlag einer vom bayerischen Wissenschaftsministerium eingesetzten Expertenkommission Aufmerksamkeit erregt, die beiden Münchner Universitäten (LMU und TU) zu einer ,University of Munich“ zu fusionieren und die bisherige Fakultätsgliederung zugunsten fachbezogener „Schools“ (man könnte hier auch von größeren „Departments“ sprechen) nach angelsächsischem Muster

20 Vgl. hierzu Ash: Bedeutet ein Abschied vom Mythos Humboldt eine „Amerikanisierung“ der deutschen Universitäten?, S. 256f.; Gebhardt: Einleitung: Jenseits von Humboldt - Humboldt?, S. 6; Daniel Fallon: Die Differenzierung amerikanischer Hochschulen nach Funktion und Bildungsauftrag, in: Breinig/Gebhardt/Ostendorf: Das deutsche und das amerikanische Hochschulsystem, S. 87-105.

21 Vgl. hierzu die Einschätzung des Präsidenten der Universität Erfurt Wolfgang Bergsdorf: Die Universität in der Wissensgesellschaft, in: Aus Politik und Zeitgeschichte B 26 (2002), S. 20-26, hier S. 23 (Zitat): „Ohne jede Frage bietet das amerikanische Hochschulwesen eine Vielzahl von wichtigen Anregungen, die für die Reform der deutschen Universitäten von erheblicher Relevanz sind. Gleichwohl läßt sich die Behauptung nicht verifizieren, daß ,die amerikanische Universität besser sei als die deutsche. Dafür ist der Standard in den USA zu unterschiedlich, der Abstand zwischen den meisten privaten Spitzenuniversitäten und den minderen Hochschulen ist dort viel breiter, als dies in Deutschland der Fall ist. Die zehn Spitzenuniversitäten allerdings sind zweifellos besser als die zehn besten deutschen Universitäten, welche Kriterien man auch immer anlegt."

22 Vgl. Mitchell G. Ash: Äpfel mit Äpfeln vergleichen! Wider die Mythen amerikanischer Universitäten in der deutschen hochschulpolitischen Diskussion, Forschung und Lehre, in: Mitteilungen des Deutschen Hochschullehrerverbandes 4 (1998), S.172-175.

23 Vgl. Weiler: Kontrollgewalt und Finanzstrukturen, S. 56-58.

24 Helmut Altner: Princeton an der Donau? Profil und Wirkungen der Regensburger Universitätsstiftung. Rede, gehalten auf der Vollversammlung der IHK am 29.5.2001 (verteiltes Redemanuskript), S.2. 
umzugestalten. Begründet wurde die vermeintliche Notwendigkeit einer solchen Hochschulfusion mit den davon zu erwartenden Synergieeffekten. Erwartungsgemäß rief der Kommissionsvorschlag bei den betroffenen Hochschulen sowie in der Öffentlichkeit ein eher geteiltes Echo hervor. Während man sich an der LMU von der Vorstellung einer Münchner „Riesenuniversität“ wenig begeistert zeigte, wurde die „Holding“-Idee von der TU-Führung durchaus positiv aufgenommen. Um mögliche Vorbehalte zu zerstreuen, bemühte TU-Präsident Wolfgang Herrmann in einem Interview mit der "Süddeutschen Zeitung" wiederum ein amerikanisches Vorbild. „Es kann doch eine große Chance sein“, so Herrmann, „wenn beide Universitäten zusammengehen. Man sollte nicht voreilig von einem Moloch reden. 1300 Professoren sind ja kein Moloch. Die beiden Physik-Fakultäten zusammen hätten die Größe derjenigen von Stanford.“25

Nur wenig später äußerte der damalige Präsident der Berliner FU, Dieter Lenzen, ebenfalls unter Verweis auf amerikanische Gegebenheiten öffentlich Zweifel an der Sinnhaftigkeit einer Münchner Hochschulfusion. Nach Lenzen stehen hinter derartigen Plänen, wie sie bereits an der Universität Duisburg-Essen umgesetzt wurden, primär Spar- und Disziplinierungsmotive des Staates und weniger der propagierte Wille zur Exzellenz auf amerikanischem Spitzenniveau:

„Ein erstes Motiv heißt: Universitäten in die Liquidation treiben. Fusionsideen im Hochschulbereich entstehen häufig aus finanziellen Engpässen - oder eine Universität, deren Leitung oder die Belegschaft ist unbequem geworden, kurz: Sie muß weg. Da man eine Universität zumindest nicht wie in Weißrußland einfach schließen und Professoren verschwinden lassen kann, läßt man sie in einer diffusen Fusionsmasse aufgehen. Gern wird dabei auf die USA verwiesen, doch dieser Hinweis ist falsch: Die US-Spitzenuniversitäten haben durchaus klassische Fakultäten, und sie haben vor allem Geld. So verfügt die Columbia Universität in New York [die Partneruniversität der FU, S. P.] dieses Jahr über 2,4 Milliarden Dollar für 19000 Studenten, die Ludwig-Maximilians-Universität in München 2003 nur über 307 Millionen Euro für 50000 Studenten. [...]. Der Effektivitätsgewinn einer University of Munich bestünde darin, daß 70000 Studenten an einer Universität studierten. Sie wäre die größte Universität der Welt, aber nicht die Beste.“26

Die mittlerweile ad acta gelegte Diskussion um eine „University of Munich“ zeigt exemplarisch, welch bizarres Ausmaß die amerikanische Vorbildfunktion im Hochschul- und Wissenschaftsbereich angenommen hat. Gegner wie Befürworter einer Amerikanisierung des deutschen Hochschulwesens verweisen auf die Verhältnisse an amerikanischen Spitzenuniversitäten, um ihr Pro oder Contra in dieser Frage zu untermauern. Mit anderen Worten: Das „Vorbild USA“ dominiert den heutigen Hochschulreformdiskurs in der Bundesrepublik in einem kaum zu überschätzenden Ausmaß. Gleichzeitig aber scheint das Bewußtsein für die historischen Voraussetzungen dieser amerikanischen Vorbildrolle nur mehr rudimentär vorhanden zu sein. Aktuelle Reformen, wie die schon erwähnte Einführung der Juniorprofessur oder die erneute Installierung von Hochschulräten, werden gegenüber der Öffentlichkeit als Novum und wichtiger Schritt zur Modernisierung

25 Angst vor der Monster-Uni - Hoffnung auf ein schützendes Dach, in: Süddeutsche Zeitung vom 16.9.2004.

26 Dieter Lenzen: Uni-Fusion: Münchner Größenwahn, in: Süddeutsche Zeitung vom 21.9. 2004. 
der Universitäten dargestellt, ohne darauf hinzuweisen, daß beinahe exakt die gleichen Reformpfade schon vor einigen Jahrzehnten mit damals eher bescheidenem Erfolg beschritten wurden.

Das eigentliche Kernproblem war und ist bis heute jedoch nicht die Frage, $o b$ wir etwas von amerikanischen Universitäten lernen können oder nicht, sondern vielmehr wie wir dies konkret tun. Nochmals: Der Transfer einzelner Elemente aus dem amerikanischen Hochschulsystem - und mögen die Motive hierfür noch so begründet scheinen - verlangt nicht nur deren Anpassung an deutsche Rahmenbedingungen, sondern in gleicher Weise auch umgekehrt die Anpassung hiesiger Strukturen an die übernommenen Elemente. Was nützt wie gesagt ein deutscher Universitätspräsident, wenn dieser nicht mit vergleichbaren Kompetenzen ausgestattet ist wie sein amerikanisches Vorbild? Oder: Was bringt ein Hochschulrat ohne wirkliches Mitspracherecht in Fragen der Universitätsverwaltung?

Auch sollte nicht vergessen werden, daß grundlegende Reformen im Hochschulbereich nicht zum Nulltarif zu bekommen sind, speziell dann nicht, wenn durch diese - wie gerne behauptet wird - das Niveau amerikanischer Spitzenuniversitäten erreicht werden soll. Der Staat, d.h. Bund wie Länder, aber auch die Hochschulen selbst müssen sich endlich darüber klar werden, wohin die Reise im 21. Jahrhundert gehen soll. Der alleinige Hinweis auf amerikanische Spitzenuniversitäten nützt nichts, solange deutsche Hochschulen nicht über vergleichbare strukturelle und finanzielle Voraussetzungen verfügen. Diesbezüglich hat auch Horst Mewes darauf hingewiesen,

„daß bedeutsame Reformen der deutschen Universitätsausbildung durch fehlende politische (und somit finanzielle) Unterstützung bisher nicht durchsetzbar waren. Wie unter diesen Umständen irgendwelche wichtigen Aspekte des generell sehr kostspieligen amerikanischen Systems auf Deutschland transferiert werden können ist unklar. [...]. Genauer gesagt ist es [das deutsche Hochschulsystem, S. P.] gezwungen, unter absolut unmöglichen Bedingungen zu arbeiten: der Unterbringung stets wachsender Studentenzahlen bei gleichzeitiger finanziellen Kürzungen, die eine kontinuierliche Reduktion personeller und materieller Ressourcen fordern. Es ist zu befürchten, daß unter derartigen Umständen die Durchsetzung ernsthafter Reformen äußerst schwierig, wenn nicht gar unmöglich ist.“27

Die im Herbst 2003 von der Bundesregierung angestoßene Diskussion um die Einrichtung von Eliteuniversitäten nach amerikanischem Muster - man spricht in diesem Zusammenhang gerne von einem oder gar mehreren „deutschen Harvard(s)“, während - wir erinnern uns - im ausgehenden 19. Jahrhundert z.B. die Johns-Hopkins-University noch voller Stolz als „Göttingen in Baltimore“ bezeichnet wurde, mutet unter den gegebenen Voraussetzungen befremdend an. ${ }^{28}$ Welche objektiven und belastbaren Kategorien sollen darüber entscheiden, ob eine Universität in den „Elite-Olymp“ aufsteigen darf oder nicht? Selbstverständlich existiert bereits heute keine wirkliche Gleichheit unter den deutschen Hochschulen. Jede Universität zwischen Kiel und Konstanz kann mit eigenen Stärken und

27 Mewes: Das amerikanische Hochschulsystem - ein Modell?, S. 196 (Zitat).

$28 \mathrm{Zu}$ der damals von der Bundesregierung angestoßenen Diskussion vgl. die folgenden höchst aufschlußreichen Artikel: Nico Fried: Von der Wiege zum Beruf, in: Süddeutsche Zeitung vom 6.1.2004; Jeanne Rubner: USA - Das große Vorbild, in: Süddeutsche Zeitung vom 15.1.2004. 
Schwächen aufwarten. Dennoch läßt sich hierzulande noch nicht - wie in den USA durchaus üblich - von Universitäten mit oder obne wissenschaftlichem Niveau reden. Faktisch aber käme der Ausbau einiger weniger Universitäten in Eliteeinrichtungen der Umwandlung der deutschen Hochschullandschaft in ein hierarchisches „Mehrklassensystem“ gleich. „Unter dem Lockruf ,Eliteuniversität" “, so warnt der Soziologe Ulrich Beck, „wird zurzeit de facto die Provinzialisierung der deutschen Universität perfektioniert. “29 Tatsächlich sollte man sich über die zu erwartenden Folgen eines derartigen Schritts im klaren sein. Es bleibt fraglich, ob der von Politik und Wirtschaft mit Nachdruck eingeforderte Wettbewerb zwischen den Hochschulen - einmal abgesehen von der Handvoll auserkorener Eliteeinrichtungen - unter derart ungleichen Rahmenbedingungen überhaupt stattfinden würde ${ }^{30}$ oder ob die künftigen Universitäten zweiter oder gar dritter Klasse nicht vielmehr gezwungen sind, sich mit ihrer Rolle abzufinden. Eine solche Entwicklung würde letztendlich wohl zu einer kaum wünschenswerten Amerikanisierung der deutschen Hochschullandschaft führen. ${ }^{31}$ „Ein paar Harvards, Stanfords und Yales“, wie Jeanne Rubner im Januar 2004 in der „Süddeutschen Zeitung“ warnte, „werden dem hiesigen Hochschulsystem nicht aus der Krise helfen. Die Oldenburgs [gemeint sind die kleineren bzw. durchschnittlichen Universitäten, S. P.] brauchen mehr Freiheit und vor allem einen verläßlicheren Staat statt all die Zaubersprüche." 32 Einen in dieser Frage völlig entgegenstehenden Standpunkt vertritt der Berliner Neurologe und damalige Vorsitzende des Wissenschaftsrates Karl Max Einhäupl, der in dem Aufbau speziell geförderter Eliteuniversitäten einen expliziten Nutzen auch für die vermeintlich „schwächeren“ Hochschulen auszumachen glaubt. In „Der Zeit“ vom 11. November 2004 schrieb Einhäupl:

„Beim Anwerben von Studierenden und Professoren muß Dresden neben Harvard bestehen können. Deutschland braucht Spitzenuniversitäten und mehr Spitzenforschung an den Universitäten. Das erfordert eine Kumulation von erstklassigen Köpfen in einer Einrichtung. [...]. Auch Hochschulen, die vielleicht nicht zur Spitzengruppe gehören werden, wollen sich um Graduiertenschulen oder Exzellenzcluster bewerben oder an solchen anderer Universitäten beteiligen. Sie alle kennen die positiven Nebenwirkungen eines solchen Wettbewerbs: Wenn einzelne Universitäten in Deutschland Weltruhm erlangen, werden alle davon profitieren. “33

Rückblickend betrachtet war es eine notwendige und ebenso richtige Entscheidung, das Hochschulwesen in den 1960er und 1970er Jahren auch mit Hilfe zahlreicher Neugründungen mit dem Ziel auszubauen, in der Bundesrepublik ein flächendeckendes und modernes Hochschulnetz zu etablieren. Der mit diesem

29 Ulrich Beck: Vorwärts zu Humboldt 2, in: Die Zeit vom 11.11.2004.

30 Vgl. exemplarisch Manfred Erhardt: Mehr Qualität und Leistung durch Wettbewerb und Eigenverantwortung. Zur Erneuerung deutscher Hochschulen, in: Aus Politik und Zeitgeschichte B 26 (2002), S. 3-6.

31 Vgl. zu dieser Debatte beispielhaft Martin Spiewak: Elite, in: Die Zeit vom 8.1.2004. Ferner siehe auch Richard Münch: Die akademische Elite. Zur sozialen Konstruktion wissenschaftlicher Excellenz, Frankfurt am Main 2007; Julia Friedrichs: Gestatten Elite. Auf den Spuren der Mächtigen von Morgen, Hamburg 2008; Heike Schmoll: Lob der Elite. Warum wir sie brauchen, München 2008.

32 Jeanne Rubner: Die Märchen-Universität, in: Süddeutsche Zeitung vom 6.1.2004.

33 Karl Max Einhäupl: Der lange Marsch zum Gipfel, in: Die Zeit vom 11.11.2004. 
Schritt eingegangenen Verantwortung sollte der Staat jedoch erst in vollem Ausmaß gerecht werden, bevor mit Blick auf Amerika über die Errichtung von Eliteuniversitäten gesprochen werden kann. Durch die faktische Reduzierung finanzieller Zuweisungen und die Streichung von Personalstellen unter dem Deckmantel der Reform wird dies bei gleichzeitig steigenden Studentenzahlen und trotz der Einführung von Studiengebühren derzeit nicht in ausreichendem Maße getan. Statt dessen läuft die von Seiten der Politik und Wirtschaft eingeforderte „Ökonomisierung“ der Hochschulen auf Kosten der Geistes-, Kultur- und Sozialwissenschaften Gefahr, die Universitäten zu rein marktorientierten Spezialhochschulen für vermeintlich verwertbarere Wissenschaftszweige zu degradieren. ${ }^{34}$ Zudem sollen die Universitäten - abermals sei hier an die Diskussionen der 1960er und 1970er Jahre erinnert - möglichst nach betriebswirtschaftlichen Gesichtspunkten wie Unternehmen geführt werden. ${ }^{35}$

Es steht selbstverständlich außer Frage, daß Hochschulen mit den ihnen zur Verfügung stehenden Geldern sinnvoll haushalten sollen und müssen. Ebenso ist es richtig, die Wirtschaft an der Finanzierung von Forschung und Lehre zu beteiligen, ja diese in eine nachhaltige Verantwortung für die weitere Entwicklung der Hochschulen zu nehmen. Dennoch sollte in diesem Zusammenhang eine schlichte Tatsache niemals außer acht gelassen werden: Universitäten sind keine Wirtschaftsunternehmen und sollten demzufolge auch nicht wie solche geführt werden. Hochschulen haben einen wichtigen und hochkomplexen gesellschaftspolitischen und kulturellen Auftrag zu erfüllen, der sich - wenn überhaupt - nur bedingt nach wirtschaftlichen Kriterien messen läßt. ${ }^{36}$ Ein derart betriebswirtschaftlich gedachter Ansatz mag in einzelnen Fächern wie Informatik oder Maschinenbau teilweise möglich sein, bei der Bewertung von Archäologen, Byzantinisten, Anglisten oder Historikern, ja selbst von Physikern und Biologen erscheint ein solches Instrumentarium weitestgehend unbrauchbar. Trotzdem verweisen die Befürworter einer „marktgerechten“ Hochschule beinahe gebetsmühlenartig auf die USA, freilich ohne gleichzeitig zu erwähnen, daß gerade die hierzulande so gepriesenen

34 Zur Diskussion um die künftige Rolle der Geisteswissenschaften an den deutschen Universitäten vgl. u.a. Wolfgang Frühwald u.a.: Geisteswissenschaften heute. Eine Denkschrift, Frankfurt am Main 1991; Walter Erhart: Amerikanisierung. Reflexionen zu einem wissenschaftspolitischen Schlagwort, in: Mitteilungen des Deutschen Germanistenverbandes 1 (2002), S. 56-72; Dieter Langewiesche: Wieviel Geisteswissenschaften braucht die Universität?, in: Kimmich/Thumfart: Universität ohne Zukunft?, S.36-51; Klaus Landfried: Die Zukunft der Universitäten und die Rolle der Geisteswissenschaften, in: ebd., S. 52-69; Dan Diner: Cultural Engineering - Oder die Zukunft der Geisteswissenschaften, in: ebd., S.70-79; Frank Meier/Uwe Schimank: Neue Steuerungsmuster an den Universitäten. Mögliche Folgen für die geisteswissenschaftliche Forschung, in: ebd., S. 97-113; Detlev Schöttker: Geisteswissenschaften im Visier des Journalismus. Zu einem Motiv des ,deutschsprachigen Feuilletons' in den neunziger Jahren, in: ebd., S. 239-254.

35 Zum Thema Universitätspräsident als Hochschulmanager aus heutiger Perspektive vgl. Peter Lundgreen: Mythos Humboldt in der Gegenwart: Lehre - Forschung - Selbstverwaltung, in: Ash: Mythos Humboldt, S.145-169, hier besonders S.164f. Vgl. auch Josef Lange: Hochschulentwicklung als inner- und außeruniversitäre Managementaufgabe, in: Hein Hoebink (Hg.): Perspektiven für die Universität 2000, Neuwied 1997, S. 63-75.

$36 \mathrm{Vgl}$. auch Dierkes/Merkens: Zur Wettbewerbsfähigkeit des Hochschulsystems in Deutschland, S. 11. 
Ivy-League-Universitäten ausgezeichnet ausgebaute Geistes-, Kultur- und Sozialwissenschaften unterhalten, die den weltweiten Ruf dieser Institutionen maßgeblich mitbegründen.

Der alleinige und nicht selten vollkommen unreflektierte Hinweis auf das „Vorbild USA“ bringt die deutsche Universität also nicht weiter. Wie in den 1960er und 1970er Jahren entlastet er allerdings vor der mühsamen Suche nach eigenständigen und damit systemimmanenten Lösungen. Vielmehr sollte endlich der Versuch unternommen werden, die zweifelsohne nicht von der Hand zu weisenden strukturellen und finanziellen Schwächen des deutschen Hochschulsystems von innen heraus zu beheben. ${ }^{37}$ So hat unter dem Eindruck der angedachten Münchner Hochschulfusion Thomas Steinfeld in der „Süddeutschen Zeitung“ zu Recht vor einem „Hochschul-Mantra“ gewarnt, das die Lösung beinahe aller Probleme auf Hochschulebene durch eine Amerikanisierung verheißt:

„Ob der Hudson bald am Karwendelgebirge entspringt? Verzweifelt versucht die Bildungspolitik, den Stand der deutschen Hochschulen auf dem internationalen Markt des Wissens zu behaupten, und je schwieriger dieses Unternehmen wird desto heller scheint eine Schrift am Horizont aufzuleuchten: ,Amerika' steht darauf geschrieben. Dort soll es Rettung geben für das System einer akademischen Bildung, das immer weniger und immer nutzloseres Wissen hervorzubringen scheint. Und weil die Vereinigten Staaten die allen Ländern überlegene Wissensmacht ist, möchte man ihr bald nach deren eigenem Muster begegnen. [...]. Die Namen der berühmten amerikanischen Universitäten dienen der deutschen Bildungspolitik mittlerweile als eine Art Mantra. Spricht man sie nur oft genug aus, so scheint man zu meinen, lasse sich ihr Geheimnis auch auf Deutschland übertragen. Wie stellt man sich das überhaupt vor? [...]. Wer die deutsche Universität wirklich amerikanisieren möchte, der muß es mit zweihundert Jahren akademischer Geschichte aufnehmen, und das ist eine unendlich größere Aufgabe als die Errichtung einer ,Holding für das neue Wissensunternehmen. “ 38

Die genaue Kenntnis der unterschiedlichen historischen Entwicklung beider Hochschulsysteme und ihrer wechselseitigen Einflüsse in den vergangenen 200 Jahren bildet somit die entscheidende Grundvoraussetzung dafür, sich über die Chancen und Risiken einer gewünschten oder befürchteten Amerikanisierung des deutschen Hochschulsystems bewußt zu werden. Die vorliegende Studie möchte hierzu ihren Beitrag leisten.

37 Vgl. Vorbild Nordamerika?, S. 15 (Zusammenfassung): „Die Konkurrenzfähigkeit der deutschen Universität bleibt erhalten, wenn sie ihre besondere Eigenart und Leistungsfähigkeit beibehält. Statt ständig nach dem, amerikanischen Vorbild‘ zu schielen, muß die Politik die qualitätvolle Weiterexistenz der deutschen Universität als eigenständige, im Einflußbereich verschiedener Kulturen stehende Bildungsinstitution im Zentrum Europas fördern."

38 Thomas Steinfeld: Das Hochschul-Mantra, in: Süddeutsche Zeitung vom 15. 9. 2004. 